\title{
Vacuum Expectation Values of Products of Chiral Currents in $3+1$ Dimensions
}

\author{
Jouko Mickelsson * \\ Center for Theoretical Physics, M.I.T., Cambridge, MA 02139, USA
}

Received June 12, 1992

\begin{abstract}
An algebraic rule is presented for computing expectation values of products of local nonabelian charge operators for fermions coupled to an external vector potential in $3+1$ space-time dimensions. The vacuum expectation value of a product of four operators is closely related to a cyclic cocycle in noncommutative geometry of Alain Connes. The relevant representation of the current is constructed using Kirillov's method of coadjoint orbits.
\end{abstract}

\section{Introduction}

In $1+1$ space-time dimensions it is known that a normal ordering of local charge operators is sufficient to make them well-defined in a suitable dense domain of a fermionic Fock space. Assuming that the physical space is compactified as the circle $S^{1}$, the normal ordered charge densities define a representation of a central extension $\widehat{L G}$ of the (Lie algebra of) the loop group $L G$ corresponding to a compact gauge group $G$. In the case of chiral fermions the central term is nontrivial and gives rise to a highest weight representation of $\widehat{L G}$. The Lie algebra of the group is an affine Kac-Moody algebra.

In higher dimensions even the normal ordered current densities do not give welldefined operators in the Fock space. Even the state created from the vacuum by an action of a typical element of the current algebra has an infinite norm. This reflects the fact that the automorphisms of the algebra of canonical anticommutation relations (CAR) generated by gauge transformations are not implementable by unitary transformations in the Fock space in space dimensions higher than one. (For a thorough discussion of CAR representations see [A].) In other words, a gauge transformation tends to take a state in the Fock space to a vector in a different Fock space corresponding to an inequivalent representation of CAR.

* Permanent address: Department of Mathematics, University of Jyväskylä, SF-40100 Jyväskylä, Finland 
One is thus lead to consider, not a representation in a single Fock space, but an action of the group of gauge transformations in a bundle of Fock spaces, parametrized by vector potentials. The representations of CAR in different fibers are in general nonequivalent.

In any case, in quantum field theory we would like to compute matrix elements like

$$
\left(\phi, X_{1} X_{2} \ldots X_{n} \psi\right),
$$

where $\phi, \psi$ are states in the quantum Hilbert space and the $X_{i}$ 's are smeared charge operators, formally

$$
X_{i}=\int_{M} J_{0}(x) f(x) d x,
$$

where $f$ is a test function, $J_{0}$ is the charge density and the integral is taken over the three dimensional physical space $M$. But how can we make sense of (1.1) if the charges are not well-defined operators in the Fock space? The answer is that the $X_{i}$ 's still make sense as sesquilinear forms in the Fock space, [R], and therefore expressions like $(\phi, X \psi)$ are meaningful when the arguments $\phi, \psi$ are restricted to a suitable dense domain $\mathscr{F}_{0} \subset \mathscr{F}$ in the Fock space. But what about expressions like $\left(\phi, X_{1} X_{2} \psi\right)$ involving products of charge operators? A sesquilinear form can be thought of as an infinite matrix but the usual product of a pair of matrices does not necessarily converge in infinite dimensions.

The convergence problem was solved by Langmann by introducing a regularized multiplication of matrices (sesquilinear forms), [L]. Instead of explaining the details of Langmann's construction I shall give a derivation of the rules how to compute the expectation values (1.1) which is based on an earlier work on gauge group actions in Fock bundles, [M1]. It will turn out that all the matrix elements of operator products $X_{1} X_{2} \ldots X_{n}$ can be evaluated using simple algebraic relations based on Lie algebra extensions generalizing the structure of an affine Lie algebra to higher dimensions.

In order to get to the bare essentials of the construction it is useful to consider a bigger group than the group $\operatorname{Map}(M, G)$ of smooth gauge transformations. To make things technically slightly simpler we assume that the physical space $M$ is a compact oriented spin manifold and that the classical spinor fields are sections of $\operatorname{Spin} \otimes E$, where Spin is the spin bundle (with fiber $\mathbf{C}^{2}$ ) and $E=M \times V$ is a trivial complex vector bundle over $M$ with a unitary representation $\varrho$ of $G$ in the fiber $V$. (The compactness of $M$ can be traded off to finiteness of a certain Sobolev norm of $g-1$, $g \in \operatorname{Map}(M, G)$, and the triviality of $E$ can be dropped if $\operatorname{Map}(M, G)$ is replaced by the group of bundle automorphisms of a principal bundle $P$ to which $E$ is an associated bundle.)

Let $H$ be the space of square-integrable classical spinor fields on $M$ (sections of Spin $\otimes E$ ) and let $H_{+}$be the subspace of $H$ spanned by vectors corresponding to the nonnegative part of the spectrum of a Dirac operator $D$, and $H_{-}$the orthogonal complement of $H_{+}$. Any linear operator $g$ in $H$ can be written as a block matrix

$$
g=\left(\begin{array}{ll}
a & b \\
c & d
\end{array}\right)
$$

with respect to the splitting $H=H_{+} \oplus H_{-}$. We denote by $G L_{p}$ the group consisting of invertible bounded linear operators in $H$ such that the off-diagonal blocks $b, c$ are in the Schatten ideal $L_{2 p}$ of operators $T$ such that $\left(T^{*} T\right)^{p}$ has a converging trace. 
It can be shown that $\operatorname{Map}(M, G) \subset G L_{2}$ when $\operatorname{dim} M=3$, [MR]. The embedding is given by the natural action of gauge transformations on spinor fields, that is, by point-wise multiplication $(T(g) \psi)(x)=\varrho(g(x)) \psi(x)$. We shall show how to compute matrix elements of products $\hat{X}_{1} \ldots \hat{X}_{n}$, where the $X_{\imath}$ 's belong to the Lie algebra of $G L_{2}$ and the hat means the corresponding second quantized operator. By restriction to the Lie algebra of the $\operatorname{subgroup} \operatorname{Map}(M, G)$ this will give all the required matrix elements of local nonabelian charges.

The quantum sesquilinear forms $\hat{X}$ form a Lie algebra $\widehat{\mathbf{g l}}_{2}$ which was derived in [MR] from a $\widehat{G L}_{2}$ action in a (dual) determinant bundle DET $_{2}^{*}$ over a Grassmannian $\mathrm{Gr}_{2}$; the latter is a homogeneous space for $G L_{2}$. Each element $W \in \mathrm{Gr}_{2}$ is a closed subspace of $H$ and thus defines a polarization $H=W \oplus W^{\perp}$. On the other hand, each polarization of $H$ defines a representation of CAR in a Fock space $\mathscr{F}_{W}$ with a Dirac vacuum $|W\rangle$ characterized by

$$
a^{*}(u)|W\rangle=0=a(v)|W\rangle \text { for } u \in W^{\perp}, v \in W,
$$

where $a^{*}(u)$ is a creation operator and $a(v)$ is an annihilaton operator with the only nonvanishing anticomutators

$$
a^{*}(u) a(v)+a(v) a^{*}(u)=(u, v) \quad u, v \in H .
$$

The inner product $(\cdot, \cdot)$ in $H$ is linear in the first and antilinear in the second argument. In the case of chiral fermions this construction is "twisted" in such a way that the bundle Vac of Dirac vacua is equal to the determinant bundle $\mathrm{DET}_{2}^{*}$, [M1]. For this reason the anomalies related to chiral gauge transformations are given in terms of gauge action in the line bundle $\mathrm{DET}_{2}^{*}$. The Schwinger terms are equal to those found in $[\mathrm{MR}]$ and were later derived in [L] using the sesquilinear approach of Ruijsenaars. In fact, there was a perturbative argument [JJ] that certain commutator anomalies would arise when quantizing a nonabelian chiral Yang-Mills-Dirac system. A geometric and mathematically consistent treatment was given in [M3, FS]. On the other hand, it has been proven by Pickrell that "normal" representations of $\widehat{G L}_{2}$ do not exist, [P1].

In Sect. 2 and 3 we shall recall some basic facts about determinant bundles over Grassmannians and group extensions $\widehat{G L}_{p}$; for more details see [MR, M2, PS]. The results on vacuum expectation values are contained in Sect. 4. In Sect. 5 we reconstruct the $\widehat{G L}_{2}$ action on vacuum line bundle from Kirillov's theory of coadjoint orbits. The appropriate orbit is the cotangent bundle $T^{*} \mathrm{Gr}_{2}$ and the prequantization line bundle for Kostant-Souriou quantization is the determinant bundle $\mathrm{DET}_{2}^{*}$ when restricted to the zero section in $T^{*} \mathrm{Gr}_{2}$.

\section{Basic Notions about Infinite-Dimensional Grassmannians}

Let $H=H_{+} \oplus H_{-}$be a polarization of an infinite-dimensional complex separable Hilbert space to a pair of closed infinite-dimensional subspaces. For any closed subspace $W \subset H$ and positive integer $p$ we denote by $\mathrm{Gr}_{p}(W)$ the Grassmannian consisting of closed subspaces $W^{\prime} \subset H$ such that

(1) the orthogonal projection $W^{\prime} \rightarrow W$ is a Fredholm operator,

(2) the projection $W^{\prime} \rightarrow W^{\perp}$ is in the Schatten ideal $L_{2 p}$.

We shall denote also $\operatorname{Gr}_{p}=G r_{p}\left(H_{+}\right)$. The Grassmannian splits to connected components $\operatorname{Gr}_{p}^{(k)}(W)$ according to the Fredholm index $k$ of the projection $W^{\prime} \rightarrow W$. 
We shall fix an orthonormal basis $\left\{e_{n}\right\}_{n \in \mathbf{Z}}$ of $H$ such that $e_{n} \in H_{+}$for $n \geq 0$ and $e_{n} \in H_{-}$for $n<0$. If $W \in \mathrm{Gr}_{p}$ then it has a basis $\left\{w_{n}\right\}_{n>0}$ (not necessarily orthonormal) such that

$$
w_{n}=\sum_{m \geq-k} \alpha_{m n} e_{m}+\sum_{m<k} \beta_{m n} e_{m}
$$

with $\alpha-1 \in L_{1}$, where $k$ is the Fredholm index of the projection $W \rightarrow H_{+}$. (In fact, it is possible to choose $w$ such that $\alpha-1$ is of finite rank.) We shall call such a $w$ an admissible basis and the set of all admissible basis is a Stiefel manifold $\mathrm{St}_{p}$.

The Stiefel manifold $\mathrm{St}_{p}$ splits to connected components $\mathrm{St}_{p}^{(k)}$ labelled by the Fredholm index $k$.

It is often convenient to think of points $W \in \mathrm{Gr}_{p}$ as operators $F: H \rightarrow H$. Namely, to each $W$ we can associate the operator $F$ such that $\left.F\right|_{W}=+1$ and the restriction of $F$ to the orthogonal complement of $W$ is -1 . Clearly $F^{2}=1$ and $F^{*}=F$. Furthermore, if we write

$$
F=\left(\begin{array}{ll}
F_{11} & F_{12} \\
F_{21} & F_{22}
\end{array}\right)
$$

with respect to the splitting $H=H_{+} \oplus H_{-}$then the off-diagonal blocks are in $L_{2 p}$, $F_{11}-1 \in L_{p}$ and $F_{22}+1 \in L_{p}$. In particular, when $W=H_{+}$then the corresponding operator $\varepsilon=F\left(H_{+}\right)$is

$$
\varepsilon=\left(\begin{array}{cc}
1 & 0 \\
0 & -1
\end{array}\right) .
$$

Differentiating the equation $F^{2}=1$ we observe that the tangent space to the Grassmannian at $F \in \mathrm{Gr}_{p}$ is represented by hermitian operators $u$ which anticommute with $F$ and such that the diagonal blocks of $u$ are in $L_{p}$ and the off-diagonal blocks in $L_{2 p}$. By Hölder inequalities, the cotangent space $T_{F}^{*} \mathrm{Gr}_{p}$ consists of hermitian operators $P$ anticommuting with $F$ and with diagonal blocks in $L_{p /(p-1)}$, off-diagonal blocks in $L_{2 p /(2 p-1)}$. The value of the linear form $P$ at $u$ is $\operatorname{tr} u P$.

We also need a generalization of the above definitions. Instead of $H_{+}$and the basis $\left\{e_{n}\right\}$ we could choose some plane $W \in \mathrm{Gr}_{p}$ with an admissible basis $w=\left\{w_{1}, w_{2}, \ldots\right\}$, complete this to a basis $\{w\}_{n \in \mathbf{Z}}$ of $H$, and define a basis $w^{\prime}$ of some $W^{\prime} \in \mathrm{Gr}_{p^{\prime}}(W)$ to be admissible relative to $w$ if the matrix $w^{\prime}(w)$ giving the projection of $w^{\prime}$ to the vectors $\left\{w_{i}\right\}_{i \geq-k}$ is of the type $1+L_{1}$, where $k$ is the Fredholm index of the projection $W^{\prime} \rightarrow W$. We shall denote the Stiefel manifold consisting of these basis $w^{\prime}$ by $\operatorname{St}_{p}(W)$. It does not depend on the choice of the admissible basis $w$ of $W$.

Let $G L_{p}$ be as in the introduction. Note that automatically the diagonal blocks of $g \in G L_{p}$ are Fredholm operators with opposite Fredholm indices. The Lie algebra $\mathbf{g l}_{p}$ consists of bounded operators in $H$ such that the off-diagonal blocks are in $L_{2 p}$. The group $G L_{p}$ acts naturally on $\mathrm{Gr}_{p}$. In fact, $\mathrm{Gr}_{p}=G L_{p} / B_{p}$, where $B_{p}$ consists of the upper triangular matrices, $c=0$.

Let $G L^{p}$ denote the group of operators $t$ (in some fixed Hilbert space) such that $t-1 \in L_{p}$. There is a natural complex line bundle, the determinant bundle DET ${ }_{p}$, over $\mathrm{Gr}_{p}$. Its fiber at $W$ consists of pairs $(w, \lambda) \in \mathrm{St}_{p} \times \mathbf{C}, w$ being a basis of $W$, with the equivalence

$$
(w t, \lambda) \sim(w, \lambda \operatorname{det} t), \quad \text { for } t \in G L^{1} .
$$


A section of the dual determinant bundle $\mathrm{DET}_{p}^{*}$ is then a function $\psi: \mathrm{St}_{p} \rightarrow \mathbf{C}$ such that $\psi(w t)=\psi(w) \cdot \operatorname{det} t$ for $t \in G L^{1}$.

\section{Some Group Actions on Bundles over Grassmannians}

There is an extension $\widehat{G L}_{p}$ of $G L_{p}$ by the abelian ideal $\operatorname{Map}\left(\mathrm{Gr}_{p}, \mathbf{C}^{\times}\right)\left(\mathbf{C}^{\times}\right.$is the multiplicative group of nonzero complex numbers) which acts in the total space of the bundle $\mathrm{DET}_{p}$. The structure of this group is explained in [MR, M2]; here we shall recall some basic facts (in slightly different way than in the references).

Define first the group $\mathscr{E}_{p}$ consisting of pairs $(g, q)$, where $g \in G L_{p}$ and $q$ is a $\infty \times \infty$-matrix valued function on $\mathrm{Gr}_{p}$ such that $g w q(W)^{-1}$ is an admissible basis of $g W$ for any admissible basis of $W$. The multiplication is defined by

$$
(g, q)\left(g^{\prime}, q^{\prime}\right)=\left(g g^{\prime}, q^{\prime \prime}\right) \quad \text { with } \quad q^{\prime \prime}(W)=q\left(g^{\prime} W\right) q^{\prime}(W) .
$$

The pairs $(1, q), q(W) \in G L^{1}$ and $\operatorname{det} q(W)=1$, for all $W \in \mathrm{Gr}_{p}$ form a normal subgroup. Dividing by this subgroup one obtains the group $\widehat{G L}_{p}$.

The action of $\widehat{G L}_{p}$ in $\mathrm{DET}_{p}$ is given by

$$
(g, q) \cdot(w, \lambda)=\left(g w q(W)^{-1}, \lambda\right),
$$

where $W$ is the plane spanned by $w$. The natural action in the space of sections of $\mathrm{DET}_{p}^{*}$ is

$$
[(g, q) \cdot \psi](w)=\psi\left(g^{-1} w q(W)\right) .
$$

The group $\widehat{G L}_{p}$ is a fiber bundle over $G L_{p}$ with fiber $\operatorname{Map}\left(\mathrm{Gr}_{p}, \mathbf{C}^{\times}\right)$. Actually, there is a group with smaller fiber which acts in $\mathrm{DET}_{p}$. In the case $p=1$ the "regularization" $q$ can be chosen to be a constant function on $\mathrm{Gr}_{1}$ and we get an extension of $G L_{1}$ by $\mathbf{C}^{\times}$; the structure of this central extension is explained in detail in [PS]. The Lie algebra $\mathbf{g l}_{1}$ is a vector space sum $\mathbf{g l}_{1} \oplus \mathbf{C}$ with the following commutators, $[\mathrm{Lu}]$,

$$
\left[(X, \lambda),\left(X^{\prime}, \lambda^{\prime}\right)\right]=\left(\left[X, X^{\prime}\right], \frac{1}{4} \operatorname{tr} \varepsilon[\varepsilon, X]\left[\varepsilon, X^{\prime}\right]\right) .
$$

When $p=2$ the extension is not central. As a vector space the Lie algebra $\widehat{\mathbf{g l}}_{2}$ is a direct sum of $\widehat{\mathbf{g l}}_{2}$ and the abelian Lie algebra of maps $h: \mathrm{Gr}_{2} \rightarrow \mathbf{C}$ of the form

$$
h(F)=\alpha+\operatorname{tr} \xi(\varepsilon-F),
$$

where $\alpha \in \mathbf{C}$ and $\xi: H \rightarrow H$ is a linear map such that the off-diagonal blocks are in $L_{4 / 3}$ and the diagonal blocks are in $L_{2}$. The commutator is defined as

$$
\left[(X, h),\left(X^{\prime}, h^{\prime}\right)\right]=\left(\left[X, X^{\prime}\right], \mathscr{C}_{X} h^{\prime}-\mathscr{L}_{X^{\prime}} h+c_{2}\left(X, X^{\prime} ; \cdot\right)\right),
$$

where $\mathscr{L}_{X}$ denotes the Lie derivative on $\mathrm{Gr}_{2}$ arising from the natural action of $G L_{2}$ on the Grassmannian and $c_{2}$ is the Lie algebra cocycle with coefficients in the space of functions (3.5), [MR],

$$
c_{2}\left(X, X^{\prime} ; F\right)=\frac{1}{8} \operatorname{tr}(\varepsilon-F)\left[[\varepsilon, X],\left[\varepsilon, X^{\prime}\right]\right] .
$$

Following Pickrell [P1] one can think of $\mathbf{g l}_{2}$ as a central extension of a Lie algebra $\mathbf{g l}_{2}^{\prime}$. As a vector space $\mathbf{g l}_{2}^{\prime}=\mathbf{g l}_{2} \oplus M_{2,4 / 3}$, where $M_{2,3 / 4}$ consists of operators 
$P: H \rightarrow H$ such that the diagonal blocks are in $L_{2}$ and the off-diagonal blocks are in $L_{4 / 3}$. The commutation relations are

$$
[(X, P),(Y, Q)]=([X, Y],[X, Q]-[Y, P]+[[\varepsilon, X],[\varepsilon, Y]]) .
$$

Thus without the last term on the right the commutator would define a semidirect product of $\mathbf{g l}_{2}$ and the abelian algebra $M_{2,4 / 3}$.

The 2-cocycle defining the central extension $\widehat{\mathbf{g l}}_{2}=\mathbf{g l}_{2}^{\prime} \oplus \mathbf{C}$ is

$$
\omega((X, P),(Y, Q))=\frac{1}{32} \operatorname{tr} \varepsilon[[\varepsilon, X],[\varepsilon, Q]]-\frac{1}{32} \operatorname{tr} \varepsilon[[\varepsilon, Y],[\varepsilon, P]] .
$$

The group $G L_{2}^{\prime}$ corresponding to $\mathbf{g l}_{2}^{\prime}$ is $G L_{2} \times M_{2,4 / 3}$ with the composition rule

$$
\begin{aligned}
\left(g_{1}, P_{1}\right) \cdot\left(g_{2}, P_{2}\right)= & \left(g_{1} g_{2}, P_{1}+g_{1} P_{2} g_{1}^{-1}+\frac{1}{2}\left[\varepsilon, g_{1} \varepsilon g_{1}^{-1}\right]\right. \\
& \left.+\frac{1}{2} g_{1}\left[\varepsilon, g_{2} \varepsilon g_{2}^{-1}\right] g_{1}^{-1}-\frac{1}{2}\left[\varepsilon, g_{1} g_{2} \varepsilon\left(g_{1} g_{2}\right)^{-1}\right]\right) .
\end{aligned}
$$

We shall later need the adjoint action of $\widehat{G L}_{2}$ on the Lie algebra $\widehat{\mathbf{g l}}_{2}$. Since the center of $\widehat{G L}_{2}$ does not contribute to the adjoint action, the action is determined by the $G L_{2}^{\prime}$ action on $\widehat{\mathbf{g l}}_{2}$. Using the commutation relations (3.6) the latter is found to be

$$
\begin{aligned}
\operatorname{Ad}_{(g, P)}(X, Q, \alpha)= & \left(g X g^{-1}, Q^{\prime}, \alpha^{\prime}\right) \text { with } \\
Q^{\prime}= & g Q g^{-1}-\left[g X g^{-1}, P\right]+\frac{1}{2}\left[g \varepsilon g^{-1},\left[g X g^{-1}, g \varepsilon g^{-1}\right]\right] \\
& +\frac{1}{2}\left[g X g^{-1},\left[g \varepsilon g^{-1}, \varepsilon\right]\right]-\frac{1}{2}\left[\varepsilon,\left[g X g^{-1}, \varepsilon\right]\right], \\
\alpha^{\prime}= & \alpha-\frac{1}{32} \operatorname{tr} \varepsilon\left[\left[\varepsilon, g X g^{-1}\right],[\varepsilon, P]\right]-\frac{1}{8} \operatorname{tr} Q\left(g^{-1} \varepsilon g-\varepsilon\right) \\
& -\frac{1}{16} \operatorname{tr} X\left(8 g^{-1} \varepsilon g-8 \varepsilon\right. \\
& \left.+\left[\varepsilon,\left[\varepsilon, g^{-1} \varepsilon g\right]\right]+\left[g^{-1} \varepsilon g,\left[\varepsilon, g^{-1} \varepsilon g\right]\right]\right) .
\end{aligned}
$$

\section{Vacuum Expectation Values of Operator Products}

To start with I shall reformulate some of the results of [PS] on representations of $\widehat{G L}_{1}$ in a language suited for a generalization to $\widehat{G L}_{2}$.

Let $w \in \mathrm{St}_{1}^{(k)}$. We define a holomorphic section $\psi_{w}$ of $D E T_{1}^{*}$ by

$$
\psi_{w}(u)=\operatorname{det} w^{*} u=\operatorname{det}\left(w_{+}^{*} u_{+}+w_{-}^{*} u_{-}\right), \quad \text { for } u \in \mathrm{St}_{1}^{(k)}
$$

and $\psi_{w}(u)=0$ otherwise. We shall think of the basis $w$ as $\mathbf{Z} \times \mathbf{N}$ matrices, the second index labels the different vectors of the basis and the first index labels the coordinates of the vectors in the standard basis $e_{n}$. The $w_{+}$part of the matrix consists of the rows labelled by nonnegative coordinate indices and $w_{-}$is the lower part consisting of rows labelled by negative indices. The blocks $w_{-}, u_{-}$are Hilbert-Schmidt matrices whereas $w_{+}-1$ and $u_{+}-1$ are of trace-class. It follows that $w^{*} u$ is of the form $1+$ a trace-class operator, and the determinant is well-defined.

An inner product in the space of finite linear combinations of the sections $\psi_{w}$ is defined by declaring

$$
\left\langle\psi_{w}, \psi_{w^{\prime}}\right\rangle=\operatorname{det}\left(w^{*} w^{\prime}\right)
$$


when both $w, w^{\prime} \in \mathrm{St}_{1}^{(k)}$ and the inner product is zero if the Fredholm indices of $w, w^{\prime}$ do not coincide. The Hilbert space completion of this inner product space is denoted by $\mathscr{F}$. It can be identified as the fermionic Fock space as follows. Any increasing sequence $\left(i_{0}, i_{1}, \ldots\right)$ of integers such that $\lim \left(i_{\mu}-\mu\right)=k$ [where the integer $k$ is called the index of the sequence (i)] defines a basis vector in $\mathscr{F}, \psi_{(i)}=\psi_{w}$ with $w=\left\{e_{i_{0}}, e_{i_{1}}, \ldots\right\}$. These vectors are orthonormal with respect to the inner product (4.2). The interpretation of $\psi_{(i)}$ is that it represents a Fock space state with holes in the negative energy sea corresponding to one-particle energy levels labelled by the negative integers in the sequence (i), and filled positive energy states labelled by the missing nonnegative integers in (i).

An element $(g, q) \in \widehat{G L}_{1}$ acts on the vectors $\psi_{w}$ by

$$
T(g, q) \psi_{w}=\psi_{g_{*} w\left(q^{*}\right)^{-1}}
$$

corresponding to the natural action $[T(g, q) \psi](u)=\psi\left(g^{-1} u q\right)$ on a general section of $\mathrm{DET}_{1}^{*}$. As already mentioned, in the case $p=1$ we may choose $q$ to be independent of $W \in \mathrm{Gr}_{1}$. This means that we can define $\widehat{G L}_{1}$ to be a central extension of $\widehat{G L}_{1}$, [PS].

An element $W \in \mathrm{Gr}_{1}$ determines the vector $\psi_{w}$ up to a phase by selecting $w$ to be an orthonormal basis of $W$. A rotation of the basis $w$ by an unitary transformation $t \in G L^{1}$ changes $\psi_{w}$ by the factor det $t^{*}$. Thus the general vacuum expectation values $\langle W|X| W\rangle:=\left\langle\psi_{w}, X \psi_{w}\right\rangle$ of some operator $X$ in $\mathscr{F}$ depends only on the point $W$ on the base manifold $\mathrm{Gr}_{1}$ and not on the choice of $w$.

The inner product $\left\langle\psi_{w}, \psi\right\rangle$, where $\psi$ is an arbitrary element of $\mathscr{F}$, can be computed in the following way. Without an essential restriction we may assume that $w$ is orthonormal. Let $\left\{f_{n}\right\}_{n \in \mathbf{Z}}$ be an orthonormal basis of $H$ such that $\left\{f_{n}\right\}_{n>0} \in \mathrm{St}_{1}^{(k)}$. For any pair $w, w^{\prime} \in \mathrm{St}_{1}^{(k)}$ we can write

$$
\operatorname{det}\left(w^{*} w^{\prime}\right)=\psi_{w}\left(w^{\prime}\right)
$$

and therefore

$$
\left\langle\phi, \psi_{w}\right\rangle=\phi(w)
$$

for any $\phi \in \mathscr{F}$. Thus we have:

Proposition 4.6. Let $W \in \mathrm{Gr}_{1}, w$ an admissible orthonormal basis of $W$, and $X \in \widehat{\mathbf{g l}}_{1}$.

$$
\langle W|X| W\rangle=\left(X \psi_{w}\right)(w)
$$

We shall now go over to the case $p=2$, corresponding to the dimension three of the physical space. In higher dimensions than one, for any given vacuum state $|W\rangle$ in the fermionic Fock space, the vector $X|W\rangle$ does not belong to $\mathscr{F}$ when $X$ is a gauge current, or a product of current components.

If $w \in \mathrm{St}_{4 / 3}$ then the formula (4.1) still defines a holomorphic section of $\mathrm{DET}_{2}^{*}$. This follows from the generalized Hölder inequalities which state that $A B$ has a finite trace if $A \in L_{p}$ and $B \in L_{q}$ with $\frac{1}{p}+\frac{1}{q}=1$. In the case at hand, $w_{-} \in L_{4 / 3}$ and $u_{-} \in L_{4}$ and so $w_{-}^{*} u_{-} \in L_{1}$; note that both $w_{+}-1$ and $u_{+}-1$ are still in $L_{1}$.

Thanks to the smooth action of $\widehat{G L}_{2}$ in $\mathrm{DET}_{2}^{*}$ the section $X \psi_{w}$ is a perfectly well-defined smooth section of the determinant bundle $\mathrm{DET}_{p}^{*}$, for any $X \in \widehat{\mathbf{g l}}_{2}$ or any 
product of elements of $\widehat{\mathbf{g l}}_{2}$, and therefore we may evaluate

$$
\left(X \psi_{w}\right)(w) \text {. }
$$

We take this expression as the definition of the vacum expectation value $\langle W|X| W\rangle$. In order to further motivate this choice, we shall show later that (4.7) is actually obtained by computing fiberwise the expectation values of the current algebra in a Fock bundle.

For any finite transformation $g \in G L_{2}$ we can compute $\left\langle H_{+}|T(g, q)| H_{+}\right\rangle$quite explicitly. The result is

$$
\left\langle H_{+}|T(g, q)| H_{+}\right\rangle=\operatorname{det}\left(a q\left(H_{+}\right)^{-1}\right),
$$

where $a=a(g)$ is as in (1.3). In particular, near the unit element in $G L_{2}$ we may choose $q\left(H_{+}\right)=a$ and then the vacuum expectation value at the true vacuum is normalized to one. Of course, this does not mean that all vacuum expectation values for elements of $\widehat{G L}_{2}$ are equal to one, but only for those elements given by the local section $G L_{2} \rightarrow \widehat{G L}_{2}$ above.

The vacuum expectation values of Fock space operators have been determined earlier by Ruijsenaars, [R]. He did not discuss the abelian extension $\widehat{G L}_{2}$. His result should be interpreted in the present context as vacuum expectation values with respect to some fixed local section $G L_{2} \rightarrow \widehat{G L}_{2}$. Recently Langmann has defined a product for the sesquilinear forms in Fock space which corresponds to the product of bundle maps discussed above, [L].

Note that the value of the spherical function $\Phi(g, q)=\left\langle H_{+}|T(g, q)| H_{+}\right\rangle$at $(g, q)$ is equal to the value of the vacuum section $\psi_{0}$ (which is defined by the basis $\left.w=w(0):=\left\{e_{0}, e_{1}, \ldots\right\}\right)$, at the point $g w q^{-1}$. Therefore all the derivatives

$$
\begin{aligned}
& \left\langle X_{1} X_{2} \ldots X_{n}\right\rangle \\
& \quad=\left.\frac{d}{d t_{1}} \ldots \frac{d}{d t_{n}} \Phi\left(e^{t_{1} X_{1}} \ldots e^{t_{n} X_{n}}(g, q)\right)\right|_{(g, q)=1, t_{1}=\ldots=t_{n}=0},
\end{aligned}
$$

which are the vacuum expectation values of the operator products $X_{1} \ldots X_{n}$, can be written

$$
\left\langle X_{1} \ldots X_{n}\right\rangle=\left(X_{1} \ldots X_{n} \psi_{0}\right)(w(0)) \text {. }
$$

The computation of the vacuum expectation values (at the free vacuum $\psi_{0}=\left|H_{+}\right\rangle$) is now completely algebraic. The vacuum $\psi_{0}$ is annihilated by all the operators $X \in \mathbf{g l}_{2} \subset \widehat{\mathbf{g l}}_{2}$ with $b(X)=0$. On the other hand, if $\psi$ is any section then

$$
(X \psi)(w(0))=0 \quad \text { when } c(X)=0 .
$$

This follows from $g^{-1} w(0) q=w(0)$ for any $g$ with $c(g)=0$ and $q=a(g)$. Thus we have the following result:

Theorem 4.12. The vacuum expectation value $\left\langle X_{1} X_{2} \ldots X_{n}\right\rangle$ of elements $X_{\imath} \in \widehat{\mathbf{g l}}_{2}$ is computed as follows. Commute all generators $\left(\begin{array}{ll}0 & b \\ 0 & 0\end{array}\right)$ to the left and all generators of the type $\left(\begin{array}{ll}a & 0 \\ c & d\end{array}\right)$ to the right, using the commutation relations (3.6). Any element on the latter type on the right gives zero when acting on the vacuum and any element of the former type on the left gives zero when it hits the vacuum on the left. We are finally 
left with some function $h$ on the Grassmannian, involving products of the cocycles $c_{2}(X, Y ; f)$ sandwiched between the vacuum states. The value of $\left\langle X_{1} \ldots X_{n}\right\rangle$ is then obtained by evaluating $h$ at the base point $F=\varepsilon$.

Corollary 4.13. $\langle X\rangle=\langle X Y\rangle=\langle X Y Z\rangle=0$ and $\langle X Y Z V\rangle=\operatorname{tr} V_{12} X_{21} Z_{12} Y_{21}+$ $\operatorname{tr} X_{21} V_{12} Y_{21} Z_{12}$ for all $X, Y, Z, V \in \mathbf{g l}_{2} \subset \widehat{\mathbf{g l}}_{2}$.

Note the difference, as compared to the case of $\widehat{\mathbf{g l}}_{1}$, already in the expectation value $\langle X Y\rangle$. For $\widehat{\mathbf{g l}}_{1}$ this is equal to $\operatorname{tr}\left(X_{21} Y_{12}\right)$ and for $X=Y^{*}$ gives the norm squared of the vector $X|0\rangle$. For $\widehat{\mathbf{g l}}_{2},\left\langle Y^{*} Y\right\rangle$ is not really a norm of any vector in the Fock space. The vacuum expectations are closely related to cyclic cocycles of a Fredholm module in Connes' noncommutative geometry, [C]. Let $X_{1}, \ldots, X_{k}$ be elements of $\mathbf{g l}_{p}$ with $k \geq 2 p$. Then the trace

$$
\tau\left(X_{1}, X_{2}, \ldots, X_{k}\right)=\frac{1}{2^{k}} \operatorname{tr} \varepsilon\left[\varepsilon, X_{1}\right]\left[\varepsilon, X_{2}\right] \ldots\left[\varepsilon, X_{k}\right]
$$

is finite and defines a cyclic cocycle in $\mathbf{g} \mathbf{l}_{p}$. It is a simple matter to show that

$$
\tau(X, Y, Z, V)+\tau(X, V, Z, Y)=\langle V Y Z X\rangle-\langle X Z V Y\rangle .
$$

I want to stress once more that we do not have a true unitary representation of the Lie algebra $\widehat{\mathbf{g l}}_{2}$ but a "pseudorepresentation" which allows to compute expectation values of products of generators compatible with the commutation relations of $\widehat{\mathbf{g l}}_{1}$ although the generators are not represented by linear operators in a Fock space. Nevertheless, the vacuum expectation values can be recovered from a group action in the Fock bundle, as will be explained below.

The bundle of Fock spaces $\mathscr{F}_{W}$ parametrized by points $W \in \mathrm{Gr}_{2}$ is constructed essentially generalizing the construction of the Fock space $\mathscr{F}=\mathscr{F}_{H_{+}}$to arbitrary position on $\mathrm{Gr}_{2}$, [M1]. Away from the base point $W=H_{+}$there is a modification of the inner product by the formula (4.2), which is related to the fact that in the case $p=2$ the admissible basis $w$ are in general nonunitary. However, in this paper we shall localize the inner products at the point $H_{+}$and therefore we leave it to the interested reader to check the general case [M1]. The dual determinant bundle $\mathrm{DET}_{2}^{*}$ over $\mathrm{Gr}_{2}$ can be thought of as a one-dimensional complex subbundle of $\mathscr{F}$, the vacuum line bundle.

Given $W \in \mathrm{Gr}_{2}$ the $\operatorname{vacuum} \operatorname{Vac}(W)$ is spanned by the section $\tilde{\phi}_{w}, \tilde{\phi}_{w}(u)=$ $\operatorname{det} u(w)$, where $w$ is an admissible basis of $W$ and the argument $u \in \operatorname{St}^{(0)}(W)$ and $u(w)$ is the matrix defining the projection of $u$ onto the vectors $w_{\imath}$. If $u \in \mathrm{St}^{(k)}(W)$ with $k \neq 0$ then we set $\tilde{\phi}_{w}(u)=0$.

The vacuum vector is well-defined up to a phase (depending on the choice of $w$ ). A section of Vac is given by $W \mapsto \phi_{W} \in \mathscr{F}_{W}$,

$$
\phi_{W}(u)=\operatorname{det}\left(w_{+}\right) \operatorname{det}(u(w)) .
$$

Note that the values of $\phi$ do not depend on the choice of $w$. The second factor in $\phi$ is invariant under the combined action of $\widehat{G L}_{2}$ on the base parameter $w$ and on the fiber variable $u$. On the other hand, the first factor is the highest weight vector in the space of sections $\Gamma\left(\mathrm{DET}_{2}^{*}\right)$. Thus $\phi$ is annihilated by all $(X, 0) \in \widehat{\mathbf{g l}}_{2}$ with $b(X)=0$. Taking into account (4.11) we get: 
Theorem 4.15. For any elements $X_{2} \in \widehat{\mathbf{g l}}_{2}$,

$$
\left\langle\phi, X_{1} X_{2} \ldots X_{n} \phi\right\rangle_{H_{+}}=\left\langle X_{1} X_{2} \ldots X_{n}\right\rangle,
$$

where $\langle\cdot, \cdot\rangle_{H_{+}}$denotes the fiber inner product at $W=H_{+}$in the Fock bundle over $\mathrm{Gr}_{2}$ and $\phi$ is the vacuum section defined by (4.14).

In Sect. 5 we shall see how the construction of $\widehat{G L}_{2}$ representations is related to Kirillov's theory of coadjoint orbits. At this point let us make some preliminary observations in relation to the vacuum expectation value computations. Let $U_{2} \subset G L_{2}$ be the unitary subgroup and $\hat{U}_{2}$ the corresponding extended group; the functions $h$ in (3.5) are then purely imaginary and $P, Q$ in (3.8) are antihermitian. If $\phi^{\prime}$ is a section of the Fock bundle which lies on the $\hat{U}_{2}$ orbit through $\phi, \phi^{\prime}=T(g, q)^{-1} \phi$, then the natural definition for the expectation values with respect to the $\phi^{\prime}$ state is

$$
\left\langle\phi^{\prime}\left|X_{1} \ldots X_{n}\right| \phi^{\prime}\right\rangle=\left\langle\phi\left|T(g, q) X_{1} \ldots X_{n} T(g, q)^{-1}\right| \phi\right\rangle .
$$

The right-hand side of (4.16) can be computed using the adjoint action of $\hat{U}_{2}$ on its Lie algebra. This is given by the formula (3.11) (for the larger group $\widehat{G L}_{2}$ ). Modulo the center, $(g, q)$ can be represented by an element $(g, P) \in G L_{2}^{\prime}$.

Restricted to the subspace $\widehat{\mathbf{g l}}_{2}$ in the enveloping algebra, the vacuum expectation values define an element in the dual space $\widehat{\mathbf{g l}}_{2}^{*}$. Using the notation at the end of the previous sectioin, a general element in $\widehat{\mathbf{g l}}_{2}$ is a triple $(X, Q, \alpha)$, where $X \in \widehat{\mathbf{g l}}_{2}, \alpha \in \mathbf{C}$, and $Q \in M_{2,4 / 3} ; Q$ represents the function $\frac{1}{8} \operatorname{tr}(\varepsilon-F) Q$ on the Grassmannian. The vacuum expectation value with respect to the $\phi$ vacuum is simply $(X, Q, \alpha) \mapsto \alpha$. By formula (3.11) the vacuum expectation value with respect to $\phi^{\prime}$ is the form

$$
\begin{aligned}
\xi(X, Q, \alpha)= & \left\langle\phi^{\prime}|(X, Q, \alpha)| \phi^{\prime}\right\rangle_{H_{+}} \\
= & \alpha-\frac{1}{32} \operatorname{tr} \varepsilon\left[\left[\varepsilon, g X g^{-1}\right],[\varepsilon, P]\right]-\frac{1}{8} \operatorname{tr} Q\left(g^{-1} \varepsilon g-\varepsilon\right) \\
& -\frac{1}{16} \operatorname{tr} X\left(8 g^{-1} \varepsilon g-8 \varepsilon-\left[\varepsilon,\left[\varepsilon, g^{-1} \varepsilon g\right]\right]\right. \\
& \left.-\left[g^{-1} \varepsilon g,\left[\varepsilon, g^{-1} \varepsilon g\right]\right]\right) .
\end{aligned}
$$

The form $\xi \in \widehat{\mathbf{g l}}_{2}^{*}$ depends on $(g, P)$ only through $G=g^{-1} \varepsilon g$ and $P^{\prime}=$ $\left[G, g^{-1} P g\right]$. The latter is a hermitian operator (for a unitary $g$ ) which anticommutes with $G$. The operator $G$ is a point on $\mathrm{Gr}_{2}$ and $P^{\prime}$ can be interpreted as a (real) cotangent vector, $P^{\prime} \in T_{G}^{*} \mathrm{Gr}_{2}, P^{\prime}(u)=\operatorname{tr} u P^{\prime}$, where $u \in T_{G} \mathrm{Gr}_{2}$. Namely, by Hölder inequalities $P^{\prime} \in M_{2,4 / 3}$; on the other hand, the diagonal blocks of $u$ are in $L_{2}$ whereas the off-diagonal blocks are in $L_{4}$. Again by Hölder inequalities the trace $\operatorname{tr} u P^{\prime}$ converges for all $u$ if and only if $P^{\prime} \in M_{2,4 / 3}$. Thus the vacua $\phi^{\prime}=T(g, q)^{-1} \phi$ (and the linear forms $\xi$ ) are parametrized by points in $T^{*} \mathrm{Gr}_{2}$.

\section{More General Matrix Elements}

Let $\mathbf{g l}_{0} \subset \mathbf{g l}_{2}$ be the subalgebra consisting of matrices $g$ in (1.3) with finite rank off-diagonal blocks. Denote

$$
\beta(X ; F)=\frac{1}{16} \operatorname{tr}[\varepsilon, F][\varepsilon, X] \text { with } X \in \mathbf{g l}_{0}, F \in \mathrm{Gr}_{2} .
$$


By a simple computation,

$$
\beta([X, Y] ; F)-\mathscr{L}_{X} \beta(Y ; F)+\mathscr{L}_{Y} \beta(X ; F)=-\frac{1}{8} \operatorname{tr} F[[\varepsilon, X],[\varepsilon, Y]]
$$

and therefore the commutation relations for the generators $\eta(X)=(X, \beta(X ; \cdot))$ [in the parametrization (3.4), (3.5)] are

$$
[\eta(X), \eta(Y)]=\left(\eta([X, Y]), \frac{1}{8} \operatorname{tr} \varepsilon[[\varepsilon, X],[\varepsilon, Y]]\right) .
$$

It follows that the generators $\eta(X)$, for $X \in \mathbf{g l}_{1}$, can be used to span a representation space for a highest weight representation of $\widehat{\mathbf{g l}}_{1}$ by acting by polynomials of the generators on the vacuum vector. Note that $\beta(X ; \varepsilon)=0$, thus the highest weight vector for $\widehat{\mathbf{g l}}_{2}$ is a highest weight vector for $\widehat{\mathbf{g l}}_{1}$.

The mixed commutation relations between the $X$ and $\eta(X)$ generators are

$$
[(X, 0), \eta(Y)]=\left([X, Y], \frac{1}{16} \operatorname{tr}[[F, X], \varepsilon][\varepsilon, Y]\right),
$$

where the cocycler converges for $X \in \mathbf{g l}_{2}, Y \in \widehat{\mathbf{g l}}_{0}$. Note that the cocycle converges actually for a slightly larger class of operators, namely for those $Y$ with $L_{4 / 3}$ offdiagonal blocks.

The subspace $\mathscr{F}_{0}$ of the Fock space consisting of finite linear combinations of vectors of finite energy and finite particle number is dense in $\mathscr{F}$. In the charge zero sector $\mathscr{F}^{(0)}$ a Fock basis can be constructed of vectors in $\mathscr{F}_{0}$ by applying the Weyl basis generators $\eta\left(E_{\imath j}\right)$ [the only nonzero matrix element of $E_{i j}$, which is equal to 1 , is in the $(i j)$ position] to the vacuum vector. The inner product of a pair of states $q|0\rangle$ and $p|0\rangle$ is $\left\langle 0\left|p^{*} q\right| 0\right\rangle$, where the antiautomorphism of the enveloping algebra of $\widehat{\mathbf{g l}}_{1}$ is determined by the hermitian conjugation of the Weyl basis generators, $\eta\left(E_{\imath j}\right)^{*}=\eta\left(E_{\jmath \imath}\right)$. Thus for each polynomial $p\left(X_{1}, \ldots, X_{n}\right)$ in the generators $X_{i} \in \mathbf{g l}_{2}$ there is a sesquilinear form

$$
\left(\psi, \psi^{\prime}\right) \mapsto\left\langle\psi|p(X)| \psi^{\prime}\right\rangle,
$$

with domain $\mathscr{F}_{0}^{(0)} \times \mathscr{F}_{0}^{(0)}$. Again, the value of the form for given pair of vectors $\psi=q(X)|0\rangle, \psi^{\prime}=q^{\prime}(X)|0\rangle$ is evaluated by shifting the raising generators to the right and lowering generators to the left and finally evaluating the cocycles at the base point $F=\varepsilon$, Theorem 4.12 .

This method works also in any highest weight representation of $\widehat{\mathbf{g l}}_{1}$, since it uses only the commutation relations and the existence of a highest weight vector. However, in the special case of the Fock representation there is a simpler way to compute matrix elements of products of generators.

Consider the associative algebra $\mathscr{B}$ which is the tensor product of the enveloping algebra of $\widehat{\mathbf{g l}}_{2}$ and the CAR algebra based on $H$, modulo the additional relations

$$
\begin{gathered}
{\left[X, a^{*}(u)\right]=a^{*}(X u), \quad[X, a(u)]=-a\left(X^{*} u\right),} \\
{\left[h, a^{*}(u)\right]=0=[h, a(u)],}
\end{gathered}
$$

where $u, v \in H, X \in \mathbf{g l}_{2}, h$ is a function on $\mathrm{Gr}_{2}$, and $X u$ means the defining action in the one-particle space.

By using the commutation relations an arbitrary element of $\mathscr{B}$ can be written as a linear combination of terms of the type

$$
R=X_{1} \ldots X_{n} h P Y_{1} \ldots Y_{k},
$$


where $P$ is an element of the CAR algebra, $h$ is a function on $\mathrm{Gr}_{2}$, the generators $X_{\imath} \in \mathbf{g l}_{2}$ have nonzero elements only in $b$-position, and the $b$-blocks of the $Y_{i}$ 's are zero. The rule for computing the vacuum expectation value of (4.24) is now the following: $\langle R\rangle=0$ if $k>0$ or $n>0$. Otherwise $\langle R\rangle$ is obtained by evaluating the function $h$ at the base point $F=\varepsilon$ and then computing $\langle P\rangle$ in the usual way using the basic canonical anticommutation relations.

\section{Matrix Elements in an Arbitrary Highest Weight Representation}

We can consider also more general highest weight pseudorepresentations. Let $\lambda=$ $\left\{\lambda_{n}\right\}_{n \in \mathbf{Z}}$ be a sequence of integers, with $\lambda_{n} \geq \lambda_{m}$ for $n>m$, such that $\lambda_{n} \rightarrow k$ when $n \rightarrow \infty$ and $\lambda_{n} \rightarrow 0$ when $n \rightarrow-\infty$, where the integer $0 \leq k$ is the level of the representation. We shall first consider a (true) representation of $\widehat{\mathbf{g l}}_{1}$, [KP]. The representation in a vector space $\mathscr{F}(\lambda)$ is characterized by a cyclic vector $|\lambda\rangle$ such that

$$
E_{\imath j}|\lambda\rangle=0 \text { for } i<j \text { and } E_{i i}|\lambda\rangle= \begin{cases}\left(\lambda_{i}-k\right)|\lambda\rangle, & \text { for } i \geq 0 \\ \lambda_{i}\left|\lambda_{i}\right\rangle & \text { for } i<0 .\end{cases}
$$

The irreducible representation of $\widehat{\mathbf{g l}}_{1}$ in the charge zero sector of the fermionic Fock space $\mathscr{F}$ is obtained in the case $k=1, \lambda_{\imath}=1$ for $i \geq 0$ and $\lambda_{i}=0$ for $i<0$.

To each $X \in \widehat{\mathbf{g l}}_{2}$ we can now associate a sesquilinear form on $\mathscr{F}(\lambda)$. Let $\mathbf{g}_{+} \subset \widehat{\mathbf{g l}}_{2}$ be the subalgebra of lower triangular matrices $g, g_{i j}=0$ for $i \geq j, \mathbf{g}_{-}$the subalgebra of upper triangular matrices, $\mathbf{h}$ the Cartan subalgebra of diagonal matrices. (Note the ordering of matrix elements; the elements with bigger indices are before those with smaller indices when reading from top to bottom or from left to right.) Any element of the enveloping algebra $\mathscr{\mathscr { C }}\left(\widehat{\mathbf{g l}}_{2}\right)$ can be written as a sum of products

$$
v=u_{-} z h u_{+},
$$

where $u_{ \pm} \in \mathscr{W}\left(\mathbf{g}_{ \pm}\right), h \in \mathscr{W}(\mathbf{h})$, and $z \in \mathscr{W}\left(\operatorname{Map}\left(\mathrm{Gr}_{2}, \mathbf{C}\right)\right)$. The vacuum expectation value of an arbitrary element in the enveloping algebra is then evaluated by a straightforward generalization of the previous rules: An element of $\mathbf{g}_{+}$acting on the vacuum on the right gives zero as well as an element of $\mathbf{g}_{-}$hitting the vacuum on the left; the vacuum is an eigenvector of $h \in \mathbf{h}$, by (4.25). After these identifications one is left with expressions $\langle z\rangle$ which are evaluated as $\langle z\rangle=z(\varepsilon)$.

\section{Interpretation in Terms of Coadjoint Orbits}

Let $G$ be a Lie group and $\mathbf{g}$ its Lie algebra. The group $G$ acts in the dual space $\mathbf{g}^{*}$ in a natural way,

$$
(g \cdot \xi)(x)=\xi\left(\operatorname{Ad}_{g^{-1}}(x)\right), \quad \text { where } x \in \mathbf{g}, \xi \in \mathbf{g}^{*}
$$

and $\operatorname{Ad}_{g}$ denotes the adjoint action of the group on its Lie algebra.

Each $G$ orbit in $\mathbf{g}^{*}$ is a symplectic manifold. The symplectic form $\omega$ is given by

$$
\omega_{\xi}(u, v)=\xi([u, v]),
$$

where tangent vectors to the orbit through the point $\xi$ ar represented by elements $u, v \in \mathbf{g}$ using the Lie algebra action on $\mathbf{g}^{*}$ [defined by left invariant vector fields, infinitesimal version of (5.1)]. 
If $\mathbf{g}$ is finite-dimensional, the dimension of the orbit $M=G \xi$ is even, say $2 n$, and the form $\omega^{n}$ defines a volume on the orbit. An orbit $M$ is quantizable if the symplectic form $\omega$ is integral, meaning that the integral of the 2 -form $\omega$ over a compact submanifold (without boundary) is always an integer. Suppose now that $M$ is quantizable. Then there is a complex line bundle $E$ over $M$ with a hermitian metric and a connection $\Gamma$ such that the curvature is equal to $\omega$. In the coadjoint method of construction group representations one first defines a Hilbert space $V=L^{2}\left(E, \omega^{n}\right)$ of square-integrable sections of the line bundle $E,[\mathrm{~K}]$.

There is an extension $\hat{G}$ of $G$ which acts in the total space of the bundle $E$, lifting the natural $G$ action on the base and preserving the hermitian metric. When $G$ is semisimple, $\hat{G}$ is the universal covering group of $G$. This action defines an unitary representation of $\hat{G}$ in $V$. In general, the representation is reducible. Sometimes irreducible representations can be obtained quite naturally if the orbit $M$ has some additional structure. A famous example is the case when $G$ is compact and semisimple and the orbit has maximal dimension (topologically, $M=G / T$ where $T$ is a maximal torus). In this case the orbit is a complex manifold, the line bundle $E$ is holomorphic and the space of holomorphic sections of $E$ carries an irreducible unitary representations of $G$; this is the Borel-Bott-Weyl theorem. In addition, the theorem tells us that all unitary irreducible representations can be obtained in this way.

The Borel-Bott-Weyl theorem has been extended to the case of an affine KacMoody group by Pressley and Segal, [PS]. However, they define the inner product in the vector space $V$ in an indirect manner. The reason is that in infinite dimensions the meaning of $\omega^{n}$ becomes problematic. In fact, up to now no suitable measure on the coadjoint orbits of an affine Kac-Moody group is known which would produce the unitary highest-weight representations.

Another infinite-dimensional example where the coadjoint orbit method has been successful (and this is the case we want to generalize) is $G=\hat{U}_{1}$, the group of unitary elements in $\widehat{G L}_{1}$. The Lie algebra, as a vector space, is now $\hat{\mathbf{u}}_{1}=\mathbf{u}_{1} \oplus i \mathbf{R}$. Consider the coadjoint orbit $M$ through the point $\xi$,

$$
\xi(x, \alpha)=-i \alpha \quad \text { with } x \in \mathbf{u}_{1} \text { and } \alpha \in i \mathbf{R} .
$$

The stability group at $\xi$ is $D \times S^{1}$, where $D \subset U_{1}$ consists of operators with zero off-diagonal blocks. The orbit $M=\hat{U}_{1} /\left(D \times S^{1}\right)$ is the Grassmannian $\mathrm{Gr}_{1}$.

Let us compute the symplectic form $\omega$ more explicitly. Let $F \in \mathrm{Gr}_{1}$ and $u, v \in T_{F} \mathrm{Gr}_{1}$. The tangent space at $F$ consists of hermitian Hilbert-Schmidt operators which anticommute with $F$. Choose $X, Y \in \mathbf{u}_{1}$ such that $u=[F, X], v=[F, X]$. This is always possible since $U_{1}$ acts transitively on $\mathrm{Gr}_{1}$. The linear form $\bar{F}$ on $\hat{\mathbf{u}}_{1}$ corresponding to $F=g^{-1} \varepsilon g$ is

$$
\bar{F}(x, \alpha)=\xi\left(\operatorname{Ad}_{g}(x, \alpha)\right)=\xi\left(x^{\prime}, \alpha-\frac{1}{2} \operatorname{tr}(F-\varepsilon) x\right)=-i \alpha+\frac{i}{2} \operatorname{tr}(F-\varepsilon) x,
$$

where $x^{\prime}$ is some element of $\mathbf{u}_{1}$. The form $\omega_{F}(u, v)$ is equal to the value of $\bar{F}$ at $[(X, 0),(Y, 0)]$ and thus

$$
\omega_{F}(u, v)=-\frac{i}{8} \operatorname{tr} \varepsilon[[\varepsilon, X],[\varepsilon, Y]]+\frac{i}{2} \operatorname{tr}(F-\varepsilon)[X, Y]=\frac{-i}{8} \operatorname{tr} F[u, v] .
$$

Since $u, v$ anticommute with $F$, this is equal to $-\frac{\imath}{4} \operatorname{tr} F u v$. 
The form $\omega$ is integral. There is a unique (up to equivalence) complex line bundle over the coadjoint orbit $\mathrm{Gr}_{1}$ with curvature $2 \pi i \omega$. In fact, this line bundle is the dual determinant bundle $\mathrm{DET}_{1}^{*}$. There is a quasi-invariant measure on the base $\mathrm{Gr}_{1}$ and the square-integrable holomorphic sections carry an irreducible representation of $\widehat{G L}_{1}$, [P2].

Let us denote by $\Lambda$ the set of pairs $(\lambda, k)$, where $\lambda=\left(\lambda_{i}\right)$ is a sequence of integers labelled by $i \in \mathbf{Z}, k$ a nonnegative integer, such that $\lambda_{\imath} \mapsto k$ when $i \mapsto+\infty, \lambda_{\imath} \mapsto 0$ when $i \mapsto-\infty$, and $\lambda_{i} \geq \lambda_{j}$ for $i>j$. For a given $\lambda$ denote by $i_{0}$ the smallest integer such that $\lambda_{i_{0}}=k$.

More general orbits in $\widehat{\mathbf{g l}}_{1}^{*}$ can now be formed by fixing $(\lambda, k) \in \Lambda$ and setting $\xi_{\lambda, k}(0, \alpha)=-i k \alpha, \xi_{\lambda, k}\left(e_{\imath i}, 0\right)=\lambda_{i}-k=0$, for $i \geq i_{0}, \xi_{\lambda, k}\left(e_{i i}, 0\right)=\lambda_{\imath}$ for $i<i_{0}$, and $\xi_{\lambda, k}\left(e_{i \jmath}, 0\right)=0$ for $i \neq j$, where $i, j \in \mathbf{Z}$. By restriction, we have also a form $\xi_{\lambda, k}: \hat{\mathbf{u}}_{1} \rightarrow \mathbf{C}$. We consider a coadjoint orbit $G(\lambda, k)$ through the point $\xi_{\lambda, k}$.

The stability group $G_{\lambda, k}$ at $\xi_{\lambda, k}$ consists of the center $S^{1}$ and of the block diagonal unitary matrices commuting with the diagonal matrix $\operatorname{diag}\left(\lambda_{\imath}\right)$. The quotient $M_{\lambda, k}=\hat{U}_{1} / G_{\lambda, k}$ is an infinite-dimensional flag manifold; in the special case when $\lambda_{i} \stackrel{1}{=} 1$ for $i \geq 0$ and $\lambda_{i}=0$ for $i<0$ we obtain the Grassmannian $\mathrm{Gr}_{1}$.

The manifold $M_{\lambda, k}$ has a natural complex structure. It can be written as a quotient of two complex groups, $M_{\lambda, k}=G L_{1} / K_{\lambda}$, where the subgroup $K_{\lambda}$ consists of matrices $g$ in $G L_{1}$ such that $g_{\imath j}=0$ for $i \leq n_{j}$, where $n_{\jmath}$ is the largest index $n$ for which $\lambda_{n}<\lambda_{\jmath}$, see the picture below.

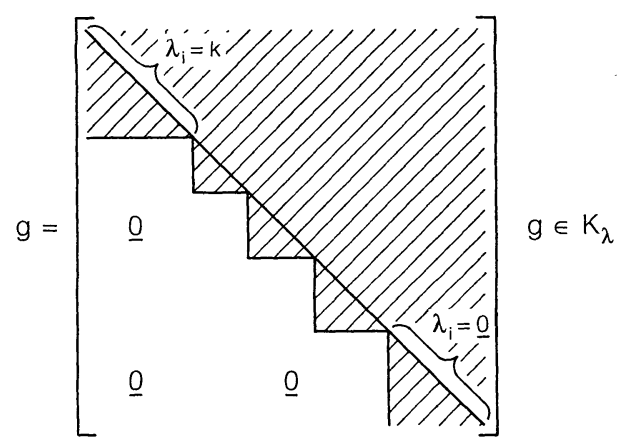

For a given weight $\lambda$ we shall denote the blocks in the stability group $G_{\lambda, k}$ by $D_{\mu}, \mu=0,1,2, \ldots, N$, where the index $\mu=0$ corresponds to the infinite block of rows and columns labelled by $i$ with $\lambda_{i}=k$, and in the other end $\mu=N$ corresponds to the infinite block of rows and columns labelled by $i$ with $\lambda_{i}=0$. The blocks in between are all of finite size. We denote by $\lambda(\mu)$ the value of the components $\lambda_{i}$ in the $\mu$ : ${ }^{\text {th }}$ block.

In the following discussion it is convenient to make a finite "vacuum subtraction." Let $\varepsilon_{0}$ be a hermitian operator in $H$ such that $\varepsilon_{0}^{2}=1$ and the difference $\varepsilon-\varepsilon_{0}$ has finite rank. Then the difference

$$
c-c_{0}=\frac{1}{8} \operatorname{tr} \varepsilon\left[[\varepsilon, X],[\varepsilon, Y]-\frac{1}{8} \operatorname{tr} \varepsilon_{0}\left[\left[\varepsilon_{0}, X\right],\left[\varepsilon_{0}, Y\right]\right]\right.
$$

of cocycles is a coboundary of the 1-cochain $\beta(X)=-\frac{1}{2} \operatorname{tr}\left(\varepsilon-\varepsilon_{0}\right) X,\left(c-c_{0}\right)(X, Y)=$ $\beta([X, Y])$. We shall now choose $\varepsilon_{0}$ to be the diagonal matrix with one's at the 
positions (ii) for $i \geq i_{0}$ and minus one's at the positions (ii) for $i<i_{0}$. The central extension $\widehat{\mathbf{g l}}_{1}$ of $\mathbf{g l}_{1}$ will be defined now with respect to $\varepsilon_{0}$ instead of $\varepsilon$.

There is a complex line bundle $\operatorname{DET}_{1}^{*}(\lambda, k)$ over $M_{\lambda, k}$ defined as follows. The sections of $\operatorname{DET}_{1}^{*}(\lambda, k)$ are complex valued functions $\psi$ on the group $\hat{U}_{1}$ such that

$$
\psi(g b)=\psi(g) \cdot \operatorname{det}\left(D_{0} q^{-1}\right) \prod_{\mu-1}^{N-1}\left(\operatorname{det} D_{\mu}\right)^{\lambda(\mu)},
$$

where $b=(D, q), D$ is a block diagonal matrix and $q$ is a matrix of the same size as $D_{0}$ such that $D_{0} q^{-1}$ has a determinant. Since left and right multiplications in a group commute, the space of functions with the characteristics (5.6) is invariant under the left action, thus defining a representation of $\hat{U}_{1}$ in the space of sections of $\operatorname{DET}_{1}^{*}(\lambda, k)$. Since the multiplication in $\widehat{G L}_{1}$ is holomorphic, the space $\Gamma_{\lambda, k}$ of holomorphic sections carries a representation of $\hat{U}_{1}$.

There is a highest weight vector $\psi_{\lambda, k}$ in $\Gamma_{\lambda, k}$, which is defined by

$$
\psi(g, q)=\prod_{\mu=0}^{N-1}\left[\operatorname{det}\left(C_{\mu}\right)\right]^{\lambda(\mu)-\lambda(\mu+1)},
$$

where $C_{\mu}$ is the infinite matrix obtained from $g q^{-1}$ by taking the rows and columns labelled by indices of the blocks $D_{0}, D_{1}, \ldots, D_{\mu}$ and $q$ is thought of as an element of $U_{1}$ by completing $q_{\imath i}=1$ for $i<i_{0}, q_{i j}=0$ when $i \neq j$ and $i<i_{0}$ or $j<i_{0}$. By elementary properties of determinants, the highest weight vector has the properties $E_{\imath \jmath} \psi_{\lambda, k}=0$ for $i<j, E_{i \imath} \psi_{\lambda, k}=0$ for $i \geq i_{0}$, and $E_{\imath i} \psi_{\lambda, k}=\lambda_{i} \psi_{\lambda, k}$ for $i<i_{0}$.

The construction above of representations of $\hat{U}_{1}$ extends to the larger group $\hat{U}_{2}$ with the following modifications. First, we should keep in mind that the operator $q$ occurring in the formulas is not a constant but a function on the Grassmannian $\mathrm{Gr}_{2}$ (with base point $\varepsilon_{0}$ ). For this reason the group action on $\operatorname{DET}_{2}^{*}(\lambda, k)$ is not holomorphic. The construction of the highest weight vector is the same as in the case of $\hat{U}_{1}$.

We started from the coadjoint orbits of the group $\hat{U}_{1}$ and noticed that the line bundles and group actions extend with the above mentioned modifications to the larger group $\hat{U}_{2}$. However, the manifolds $U_{2} / K_{\lambda}$ are not coadjoint orbits of $\hat{U}_{2}$. Because of the larger normal subgroup in the extension, the coadjoint orbits of $\hat{U}_{2}$ have roughly speaking twice the dimension of the coadjoint orbits $\hat{U}_{1}$. To illustrate this point we consider the case of the orbit $\mathrm{Gr}_{1}$ as an example.

Let $\xi \in \widehat{\mathbf{g l}}_{2}^{*}$ be the linear form defined by $\xi(X, Q, \alpha)=-i \alpha$, where we are using the parametrization at the end of Sect. 3, $X \in \mathbf{g l}_{2}, Q \in M_{2,4 / 3}$, and $\alpha \in \mathbf{C}$. That is, $i \xi(X, Q, \alpha)$ is the vacuum expectation value of $(X, Q, \alpha)$ with respect to the free vacuum. Using (3.11) we conclude that in the coadjoint action by an element $(g, P)^{-1} \in G L_{2}^{\prime}$ (we are again dropping the center of $\widehat{G L}_{2}$ since it does not contribute to the (co)adjoint action) the form $\xi$ is transported to the form $\xi_{g, P}$, given by

$$
\begin{aligned}
i \xi_{g, P}(X, Q, \alpha)= & \alpha-\frac{1}{32} \operatorname{tr} \varepsilon\left[\left[\varepsilon, g X g^{-1}\right],[\varepsilon, P]\right]-\frac{1}{8} \operatorname{tr} Q\left(g^{-1} \varepsilon g-\varepsilon\right) \\
& -\frac{1}{16} \operatorname{tr} X\left(8 g^{-1} \varepsilon g-8 \varepsilon-\left[\varepsilon,\left[\varepsilon, g^{-1} \varepsilon g\right]\right]\right. \\
& \left.-\left[g^{-1} \varepsilon g,\left[\varepsilon, g^{-1} \varepsilon g\right]\right]\right) .
\end{aligned}
$$


As we noted in Sect. 4, this form can be interpreted as the vacuum expectation value of $(X, Q, \alpha)$ with respect to a vacuum on the $\hat{U}_{2}$ orbit through the free vacuum. We also saw that the forms $\xi_{g, P}$ depend only on $G^{2}=g^{-1} \varepsilon g$ and $\left[G, g^{-1} P g\right]$, the latter operator being a vector in $T_{G}^{*} \mathrm{Gr}_{2}$. Thus the coadjoint orbit through $\xi$ can be identified as the cotangent bundle $T^{*} \mathrm{Gr}_{2}$.

The stability group of $\xi$ in $U_{2}^{\prime}$ is the semidirect product of $D$ and the abelian group $A$ of elements $(1, P)$, where $P \in M_{2,4 / 3}$ commutes with $\varepsilon$. The quotient manifold $\hat{U}_{2} /(D \ltimes A)$ is the cotangent bundle $T^{*} \mathrm{Gr}_{2}$. The $\hat{U}_{2}$ action on $T^{*} \mathrm{Gr}_{2}$ can be described as follows. Let $P \in T_{G}^{*} \mathrm{Gr}_{2}$ and $(h, Q) \in U_{2}^{\prime}$. Then

$$
\begin{aligned}
(h, Q) \cdot(G, P)= & \left(h G h^{-1}, h P h^{-1}+\left[h G h^{-1}, Q\right]\right. \\
& \left.+\frac{1}{2}\left[\left[\varepsilon, h G h^{-1}-h \varepsilon h^{-1}\right], h G h^{-1}\right]-\frac{1}{2} h[[\varepsilon, G], G] h^{-1}\right) .
\end{aligned}
$$

Let $(\delta G, \delta P)$ be a tangent vector to $T^{*} \mathrm{Gr}_{2}$ at the base point $(G, P)$. Then from (5.9) one can reduce that the vector field on $T^{*} \mathrm{Gr}_{2}$ generated by the element $\left(\frac{1}{2} \delta G G, \frac{1}{4}[\varepsilon-G,[\varepsilon, \delta G G]]+\frac{1}{4}[G, \delta P]\right)$ in the Lie algebra $\mathbf{g l}_{2}^{\prime}$, when evaluated at $(G, P)$, is equal to $(\delta G, \delta P)$. Using the commutation relations (3.8)-(3.9) and inserting to the formula (5.8) one obtains, after a bit tedious but completely straightforward algebra, the following formula for the Kirillov form on the coadjoint orbit $T^{*} \mathrm{Gr}_{2}$ :

$$
\begin{aligned}
\Omega_{(G, P)}\left(\left(\delta_{1} G, \delta_{1} P\right),\left(\delta_{2} G, \delta_{2} P\right)\right)= & \frac{i}{32} \operatorname{tr} G\left(\left[\delta_{2} P, \delta_{1} G\right]-\left[\delta_{1} P, \delta_{2} G\right]\right) \\
& -\frac{i}{32} \operatorname{tr}(\varepsilon-G)\left[\delta_{1} G, \delta_{2} G\right] .
\end{aligned}
$$

The first term on the right is the canonical 2-form on a cotangent bundle whereas the second term (which depends only on data on the Grassmannian) is a generator of the second cohomology on $\mathrm{Gr}_{2}$, [Q].

To each element $X \in \hat{u}_{2}$ one can associate the Hamiltonian function $f_{X}: T^{*} \mathrm{Gr}_{2} \rightarrow$ $\mathbf{R}$ (or by complexification of the Lie algebra, a complex function $f_{X}$ for any $X \in \widehat{\mathbf{g l}}_{2}$ ), defined by

$$
f_{X}(G, P)=\xi_{G, P}(X)
$$

It satisfies

$$
\mathscr{L}_{X} \cdot f_{Y}=f_{[X, Y]}=\Omega\left(\mathscr{L}_{X}, \mathscr{L}_{Y}\right)
$$

where we have denoted by $\mathscr{E}_{X}$ the vector field on the coadjoint orbit $M=T^{*} \mathrm{Gr}_{2}$ generated by left translations by $X$. Denote by $\{\cdot\}$ the Poisson brackets determined by the symplectic form $\Omega$. Because of (5.12),

$$
\left\{f_{X}, f_{Y}\right\}=f_{[X, Y]} \text {. }
$$

In geometric quantization one associates an operator $\hat{f}$ to each element $f \in$ $C^{\infty}(M)$. Choosing a complex line bundle $E$ over $M$ with connection $\nabla$ such that the curvature of $\nabla$ is equal to $2 \pi i \Omega$ the quantum operator $\hat{f}$ for any $f \in C^{\infty}(M)$ acts on sections of $E$ and is given by

$$
\hat{f}=\frac{1}{2 \pi} \nabla_{V_{f}}-i f
$$


where $V_{f}$ is the Hamiltonian vector field corresponding to $f, V^{\prime} \cdot f=\Omega\left(V^{\prime}, V_{f}\right)$ for any vector field $V^{\prime}$.

The above general construction gives a representation of $\widehat{\mathbf{g l}}_{2}$ on sections of $E$. For any $X \in \widehat{\mathbf{g l}}_{2}$ one can associate an operator $\hat{X}$ by

$$
\hat{X}=\frac{1}{2 \pi} \nabla_{X}-i f_{X}
$$

where for brevity we denote $\nabla_{X}=\nabla_{\mathscr{L}_{X}}$, the covariant derivative along the vector field generated by the Lie algebra action on $M$.

Proposition 5.16. The formula (5.15) defines a faithful representation of $\widehat{\mathbf{g l}}_{2}$ in the space of sections $\Gamma(E)$.

Proof. The group $U_{2}^{\prime}$ acts clearly effectively on $T^{*} \mathrm{Gr}_{2}$ and therefore to prove that the representation is faithful we have to check only that the center of $\widehat{\mathbf{g l}}_{2}$ is represented nontrivially. The Hamiltonian function $f_{c}=f_{(0,0, i)}$ corresponding to the central Lie algebra element $c=(0,0, i)$ is the constant function $f_{c}(G, P)=1$. The corresponding Hamiltonian vector field is then identically zero and so the quantum operator is the multiplication operator

$$
\hat{f}=-i f_{c}=-i
$$

By (5.10) the symplectic form is a sum of two pieces $\Omega_{0}$ and $d \theta$, where the latter is the canonical exact 2 -form on a cotangent bundle and the former is nonvanishing only along $\mathrm{Gr}_{2}$. The pull-back of $\Omega_{0}$ on $\mathrm{Gr}_{2}$ (with respect to the zero section $\mathrm{Gr}_{2} \rightarrow T^{*} \mathrm{Gr}_{2}$ ) is the curvature of the determinant bundle $\mathrm{DET}_{2}^{*}$ divided by $2 \pi i$. Thus we may think of $E$ as the pull-back of $\mathrm{DET}_{2}^{*}$ on $T^{*} \mathrm{Gr}_{2}$.

The natural polarization on the cotangent bundle leads to Schrödinger picture of quantization of the Hamiltonian functions $f_{X}$.

Theorem 5.18. The space of sections of $E$ which are covariantly constant along the fibers of $T^{*} \mathrm{Gr}_{2}$ (and can be identified as sections of $\mathrm{DET}_{2}^{*}$ ) is invariant under the $\widehat{\mathbf{g l}}_{2}$ action and so gives representation of $\widehat{\mathbf{g l}}_{2}$ in the space $\Gamma\left(\mathrm{DET}_{2}^{*}\right)$.

Proof. Denoting by $A$ a (local) potential for the curvature $2 \pi i \Omega_{0}$, the quantum operators can be written as

$$
\hat{X}=\frac{1}{2 \pi} \mathscr{L}_{X}+\frac{1}{2 \pi} A(X)+i \theta(X)-i f_{X}
$$

From (5.8) one can check that $\theta(X)-f_{X}$ is a function of $G$ only for any $X \in \widehat{\mathbf{g l}}_{2}$. Since the local potential $A$ is also a function only on the base $\mathrm{Gr}_{2}$, the operators $\hat{X}$ are of the type $\frac{1}{2 \pi} \mathscr{L}_{X}+a$ multiplication operator by a function on $\mathrm{Gr}_{2}$. The sections $\psi$ constant along fibers of $T^{*} \mathrm{Gr}_{2}$ are characterized by $\nabla_{(0, Q, 0)} \psi=\mathscr{S}_{(0, Q, 0)} \psi=0$, for $Q \in M_{2,4 / 3}$. By the remark above, this property is preserved under the action by the operators (5.19). 


\section{References}

[A] Araki, H.: Bogoliubov automorphisms and Fock representations of canonical anticommutation relations. In: Contemporary mathematics. American Mathematical Society, Vol. 62 (1987)

[C] Connes, A.: Noncommutative differential geometry. Publ. Math. IHES 62, 81 (1986)

[FS] Faddeev, L., Shatasvili, S.: Theor. Math. Phys. 60, 770 (1984)

[JJ] Jackiw, R., Johnson, K.: Anomalies of the axial vector current. Phys. Rev. 182, 1459 (1969)

[K] Kirillov, A.A.: Elements of the theory of representations. Berlin, Heidelberg, New York: Springer 1976

[KP] Kac, V., Peterson, D.: Lectures on infinite wedge representations and MKP hierarchy. Seminaire de Math. Sup. 102, 141, Montreal University (1986)

[L] Langmann, E.: Fermion and boson current algebras in 3+1 dimensions. In: Proceedings of the Symposium, Topological and Geometrical Methods in Field Theory. Mickelsson, J., Pekonen, O. (eds.). Singapore: World Scientific Publ. 1992

[Lu] Lundberg, L.-E.: Quasi-free second quantization. Commun. Math. Phys. 50, 103 (1976)

[M1] Mickelsson, J.: Commutator anomaly and the Fock bundle. Commun. Math. Phys. 127, 285 (1990); On the Hamiltonian approach to commutator anomalies in $3+1$ dimensions. Phys. Lett. B 241, 70 (1990)

[M2] Mickelsson, J.: Current algebras and groups. New York, London: Plenum Press 1989

[M3] Mickelsson, J.: Chiral anomalies in even and odd dimensions. Commun. Math. Phys. 97, 361 (1985)

[MR] Mickelsson, J., Rajeev, S.: Current algebras in $d+1$ dimensions and determinant bundles over infinite-dimensional Grassmannians. Commun. Math. Phys. 116, 365 (1988)

[P1] Pickrell, D.: On the Mickelsson-Faddeev extension and unitary representations. Commun. Math. Phys. 123, 617 (1989)

[P2] Pickrell, D.: Measures on infinite-dimensional Grassmann manifolds. J. Funct. Anal. 70, 323 (1987)

[PS] Pressley, A., Segal, G.: Loop groups. Oxford: Clarendon Press 1986

[Q] Quillen, D.: Superconnection character forms and the Cayley transform. Topology 27, 211 (1988)

[R] Ruijsenaars, S.N.M.: On Bogoliubov transformations for systems of relativistic charged particles. J. Math. Phys. 18, 517 (1977). Index formulas for generalized Wiener-Hopf operators and boson-fermion correspondence in $2 N$ dimensions. Commun. Math. Phys. 124, 553 (1989)

Communicated by K. Gawedzki 